\title{
LincRNA-p21 activates endoplasmic reticulum stress and inhibits hepatocellular carcinoma
}

\author{
Yang Ning ${ }^{1, *}$, Fu Yong ${ }^{1, *}$, Zhang Haibin ${ }^{1}$, Sima Hui ${ }^{1}$, Zhu Nan ${ }^{1}$, Yang Guangshun ${ }^{1}$ \\ ${ }^{1}$ Hepatobiliary Surgery Department V, Eastern Hepatobiliary Surgery Hospital, Second Military Medical University, Shanghai, \\ China \\ *These authors have contributed equally to this work
}

Correspondence to:

Yang Guangshun, e-mail: yangguangshunlab@sina.com

Keywords: lincRNA-p21, hepatocellular carcinoma, ER stress, sorafenib

Received: April 18, 2015 Accepted: July 08, $2015 \quad$ Published: July 20, 2015

\section{ABSTRACT}

LincRNA-p21 is a downstream long non-coding RNA (IncRNA) transcript of p53. LincRNA-p21 serves as a repressor in p53-dependent transcriptional responses and participates in diverse biological processes, including apoptosis, cell cycle, metabolism and pluripotency. However, the role of lincRNA-p21 in human hepatocellular carcinoma remains to be defined. Here in this work, we demonstrated that lincRNA-p21 acted as a tumor suppressive IncRNA in human hepatocellular carcinoma. We firstly found the downregulation of lincRNA-p21 level in human hepatocellular carcinoma tissues, and showed that low expression of lincRNA-p21 was associated with high disease stage and predicted poor survival. Further we showed that lincRNA-p21 knockdown promoted proliferation and colony formation of HepG2, Huh7 and Bel-7042 cells in vitro, while lincRNA-p21 overexpression obtained oppose results. Using tumor xenograft experiments, we also demonstrated that lincRNA-p21 inhibited HepG2 cell growth in vivo and lincRNA-p21 contributed to sorafenib-induced growth regression of HepG2 cell in vivo. Further mechanism analysis revealed that lincRNA-p21 promoted ER stress both in vitro and in vivo, which facilitated apoptosis of hepatocellular carcinoma cells. Finally, we demonstrated that ER stress accounted for lincRNA-p21 effects on apoptosis, proliferation and in vivo growth of hepatocellular carcinoma. These findings implicate that lincRNA-p21 is a potential prognostic factor and therapeutic target for human hepatocellular carcinoma.

\section{INTRODUCTION}

Hepatocellular carcinoma (HCC) causes more than 500,000 deaths each year worldwide [1]. However, measures aiming at preventing $\mathrm{HCC}$ development in these patients are limited [2]. In addition, current therapies for HCC often obtain poor long-term outcome due to drug resistance with elusive mechanisms [3]. These facts prompt us to identify novel molecular mechanisms for hepatocellular carcinoma development and drug resistance.

Endoplasmic reticulum (ER) stress has emerged as a major site of cellular homeostasis regulation, particularly in the unfolded protein response, which plays a major role in cancer and many other diseases [4]. Several drugs that activate ER stress have been approved for preclinical and clinical use. These drugs include sorafenib, eeyarestatin,
17-AAG, radicicol, and MAL3-101 [5]. The contribution of ER stress to HCC has been proposed repeatedly [6, 7], however, high activation of ER stress by sorafenib could induce apoptosis and growth arrest in established or advanced HCC $[8,9]$. Similar situation was observed for marine prostanoid, curcumin, and genistein [10-12]. The mechanism by which sorafenib induces ER stress and HCC cell apoptosis is still elusive.

Long non-coding RNAs (lncRNAs) have recently been found to be pervasively transcribed in the human genome. Aberrant expression of several lncRNAs was found to be involved in the carcinogenesis of human HCC $[13,14]$. The p53-regulated long noncoding RNA lincRNA-p2 1 has been proposed to act in trans via several mechanisms ranging from repressing genes in the p53 transcriptional network to regulating mRNA 
translation and protein stability [15]. The physiological and pathological functions of lincRNA-p21 were identified gradually. For instance, Yang et al. [16] reported lincRNA-p21 as a regulator for the Warburg effect and also implicated lincRNA-p21 as a valuable therapeutic target for cancer. Wu et al. [17] showed that lincRNA-p21 regulated neointima formation, vascular smooth muscle cell proliferation, apoptosis, and atherosclerosis by enhancing p53 activity. LincRNA-p21 function in pluripotency was also been identified $[18,19]$. Here in the present work, we identified lincRNA-p21 as a negative regulator for HCC development and drug resistance.

\section{RESULTS}

\section{LincRNA-p21 is down-regulated in human hepatocellular carcinoma}

To investigate the potential functions of lincRNA-p21 in human hepatocellular carcinoma (HCC), we firstly tested the expression profile of this lincRNA in normal liver tissues and tissues from HCC. The results showed that lincRNA-p21 level was significantly reduced in tissues from HCC than that from normal liver tissues (Figure 1A). We also tested the expression of other lncRNAs (HULC, H19, MALAT-1, TUC338, and HOTTIP) that are reported in HCC [20-24]. All of these lncRNAs were up-regulated in HCC (Supplementary Figure S1), which was different from lincRNA-p21. Therefore, we focused on lincRNA-p21 in the present work. The expression of lincRNA-p21 in tumor and adjacent tissues was also analyzed. Markedly, lincRNA-p21 level in tumor tissues was lower than that of adjacent tissues (Figure 1B). We also found the low level of lincRNA-p21 in liver cancer cell lines compared to normal human hepatocytes (Figure 1C). Further, we investigated whether lincRNA-p21 was correlated with clinical markers for HCC. We analyzed the serum levels of alphafetoprotein (AFP) in normal donors and HCC patients, and found the high levels of AFP in patients with HCC (Figure 1D). Linear regression analysis implicated the significant but negative correlation between serum AFP level and tissue lincRNA-p21 level (Figure 1E). These data indicate the potential participation of lincRNA-p21 in human hepatocellular carcinoma. To further confirm this correlation, we analyzed whether lincRNA-p21 predicted patients' survival. The results showed that low lincRNA-p21 predicted poor overall and diseasefree survival in patients with $\mathrm{HCC}$ (Figure 2A and 2B). In addition, we found that low level of lincRNA-p21 was associated with higher tumor grade $(p=0.0166)$ and stage $(p=0.0023)$, and vascular invasion $(p=0.0209 ;$ Table 1).

\section{LincRNA-p21 inhibits hepatocarcinoma cell proliferation and colony formation}

The above findings strongly implicated the participation of lincRNA-p21 in human HCC, which prompted us to study the functions of lincRNA-p21 in HCC. We first performed experiments to investigate whether lincRNA-p21 could affect cellular behaviors of liver cancer cells in vitro. We knocked down lincRNA-p21 by lentivirus-mediated shRNAs in liver cancer cell lines (Figure 3A and Supplementary Figure S2). Four shRNAs were tested and the first and third shRNAs were selected for further investigation for their relative high knockdown efficiency (Figure 3A). We found that lincRNA-p21 knockdown promoted the proliferation of HepG2 cells (Figure 3B and Supplementary Figure S3A and S3B). LincRNA-p21 knockdown also facilitated proliferation of Huh7 and Bel-7042 cells (Figure 3C and 3D). Further, we investigated whether lincRNA-p21 regulated cellular colony formation of liver cancer cells. The results demonstrated the negative effects of lincRNA-p21 on colony formation of HepG2 (Figure 3E and Supplementary Figure S3C), Huh7 (Figure 3F) and Bel-7042 (Figure 3G) cells. We also overexpressed lincRNA-p21 in liver cancer cells and found that lincRNA-p21 overexpression inhibited proliferation and cellular colony formation of HepG2, Huh7 and Bel-7042 cells (Figure 4A-4G). These findings indicate that lincRNA-p21 inhibits liver cancer cell proliferation and colony formation.

\section{LincRNA-p21 inhibits liver cancer cell growth and drug resistance in vivo}

We next studied whether lincRNA-p21 also regulated in vivo growth of liver cancer cells. We performed tumor xenograft experiments and knocked down lincRNA-p21. LincRNA-p21 knockdown did not induce any toxicity in mice. We found that lincRNA-p21 knockdown facilitated the in vivo growth of HepG2 cells (Figure 5A and 5B). In consistence, lincRNA-p21 overexpression inhibited growth of HepG2 cells in vivo (Figure 5C and 5D). These results demonstrated lincRNA-p21 acted as a negative regulator for liver cancer cell growth in vivo. Another question we were interested was whether low lincRNA-p21 contributed to drug resistance. Therefore, we used the clinical drug sorafenib, which was reported to induce apoptosis of liver cancer cells partly through inducing ER stress [8, 25]. Firstly, we found that sorafenib could induce the expression of lincRNA-p21 in vitro and in vivo (Supplementary Figure S4A and S4B). Sorafenib treatment did not induce any toxicity in mice but inhibited in vivo growth of HepG2 cells significantly. However, when lincRNA-p21 was knocked down, sorafenib was unable to repress tumor growth (Figure 5E-5F).These findings implicated that lincRNA-p21 downregulation may contribute to clinical tumor growth and drug resistance. 
A

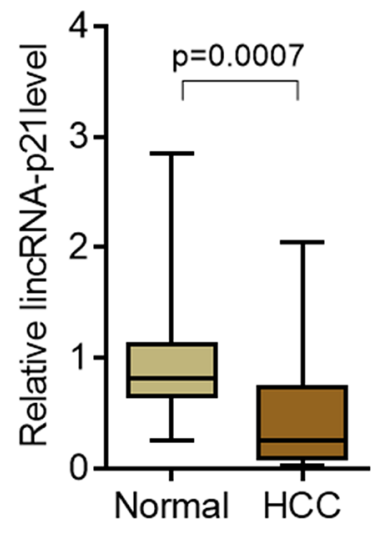

$\mathrm{D}$

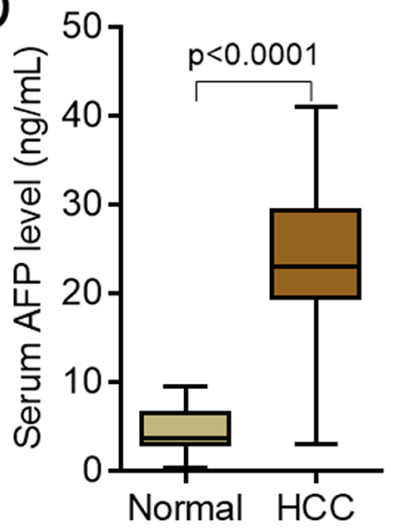

B

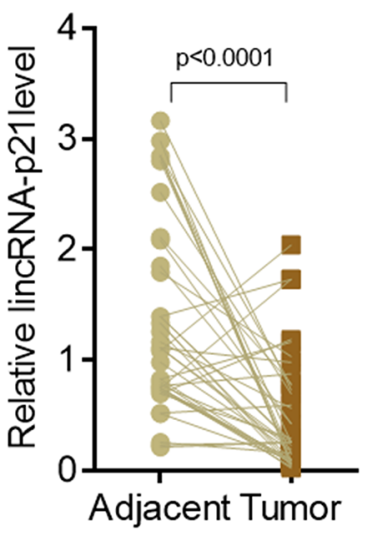

$\mathrm{E}$

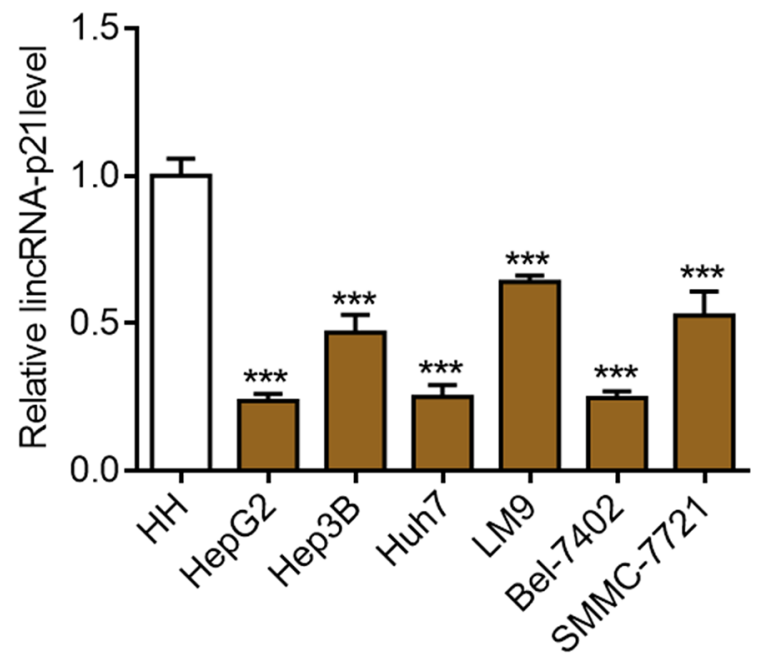

Figure 1: LincRNA-p21 is down-regulated in hepatocellular carcinoma. A. LincRNA-p21 expression in human hepatocellular carcinoma (HCC, $n=42$ ) or control normal liver tissues $(n=22)$. B. LincRNA-p21 levels in adjacent normal liver tissues and tumor tissues in HCC. $n=40$. Paired Student's $t$ test was performed to analyze the data. C. LincRNA-p21 expression in normal human hepatocytes $(\mathrm{HH})$ and liver cancer cell lines (HepG2, Hep3B, Huh7, LM9, Bel-7042, SMMC-7721). ${ }^{* * *} p<0.001$ vs. HH D. Serum levels of alphafetoprotein (AFP) in HCC patients $(n=42)$ or normal tissue donors $(n=24)$. E. Correlation between serum AFP and lincRNA-p21 level $(n=42)$

\section{LincRNA-p21 induces ER stress in hepatocellular carcinoma}

Induction of ER stress is one of the important pathways for clinical drugs to induce liver cancer cell apoptosis and repress carcinoma development $[8,10,11$, 26]. Sorafenib is a small molecule that inhibits tumorcell proliferation and tumor angiogenesis and increases the rate of apoptosis in a wide range of tumor models. In preclinical experiments, sorafenib has anti-proliferative activity in liver-cancer cell lines, and it reduces tumor angiogenesis and tumor-cell signaling and increases tumorcell apoptosis in a mouse xenograft model of human HCC [27]. One of the major mechanisms by which sorafenib inhibits liver cancer survival is by inducing ER stress $[8$, 9]. As we showed the contribution of lincRNA-p21 to sorafenib-induced repression of tumor growth, we next wanted to know whether this contribution depended upon ER stress. We analyzed ER stress markers and found the positive correlation between lincRNA-p21 levels and ER stress markers (IRE1, CHOP, and GRP78) in tissues from HCC (Figure 6A-6C). In HepG2 cells, overexpression of lincRNA-p21 induced expression of IRE1, CHOP and GRP78 (Figure 6D). We also found the upregulation of IRE, CHOP and GRP78 protein level and hyper phosphorylation of eIF2 $\alpha$ in HepG2 cells overexpressed with lincRNA-p21 (Figure 6E and Supplementary Figure S5). We also tested the activation of protein kinase RNA-like endoplasmic reticulum kinase (PERK), which is responsible for phosphorylation of initiation factor eIF $2 \alpha$, We found that lincRNA-p21 could up-regulate the phosphorylation level of PERK (Supplementary Figure S6). Finally, we studied the effect of lincRNA-p21 on ER stress in vivo. In consistent with the in vitro findings, lincRNA-p21 overexpression 
A

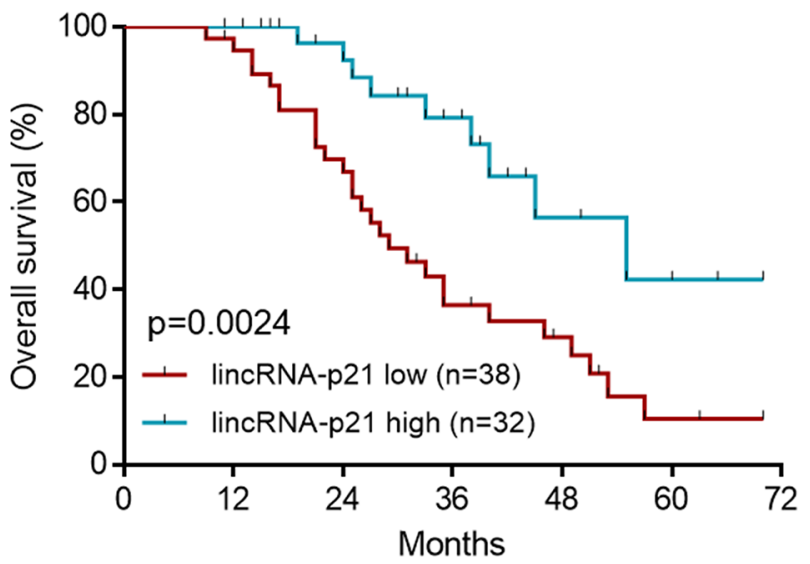

B

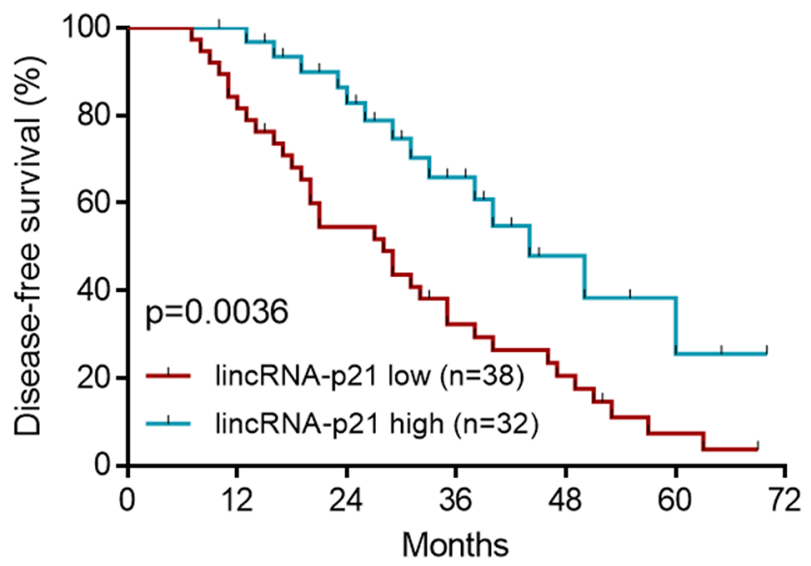

Figure 2: LincRNA-p21 low expression predicts poor survival. The patients were divided to lincRNA-p21 high group and lincRNA-p21 low group by the mean value of total 70 HCC patients. A. Low lincRNA-p21 level predicts poor overall survival in HCC patients. B. Low lincRNA-p21 predicts poor disease-free survival in HCC patients.

activated ER stress by up-regulating the transcription of ER stress markers (Figure 6F-6G and Supplementary Figure S7). In addition, as reported by previous findings, sorafenib induced ER stress in vivo (Figure 7A-7D) and in liver cancer cell lines (Figure 7E-7G). Interestingly, lincRNA-p21 knockdown blocked sorafenib effects on ER stress both in vivo (Figure 7A-7D and Supplementary Figure S8A) and in vitro (Figure 7E-7G and Supplementary Figure S8B-S8D), indicating that lincRNA-p21 contributes to sorafenib-induced ER stress.

\section{LincRNA-p21 induces apoptosis of hepatocellular carcinoma cells}

High level of ER stress can lead to apoptosis of liver cancer cells [8], therefore, we next studied whether lincRNA-p21 induced HCC cell apoptosis. We found that lincRNA-p21 overexpression induced apoptosis of HepG2 cells (Figure 8A and 8B). Similar results were observed in Huh7 and Bel-7042 cells (Figure 8B). What's more, we showed that lincRNA-p21 knockdown blocked sorafenib-induced apoptosis of HepG2 cells (Figure 8C). As lincRNA-p21 was reported to regulate p53 downstream genes expression [17], we also investigated whether lincRNA-p21 affected these genes expression in hepatocellular carcinoma. We found that lincRNA-p21 knockdown inhibited sorafenib-induced expression of p53 downstream genes, including Bax, Puma, Mdm2, and Noxa (Figure 8D-8G). These findings indicated that lincRNA-p21 contributed to sorafenib-induced apoptosis. ER stress induced apoptosis is mainly mediated through reactive oxygen species (ROS). Therefore, we tested whether lincRNA-p21 regulates cellular ROS level. We found that that lincRNA-p21 overexpression could significantly up-regulated total ROS level ( $\sim 3.4$ fold, Supplementary Figure S9). In addition, lincRNA-p21 also up-regulated the expression of dual oxidase 1 (DUOX1,
Supplementary Figure S9), which is the main source of ROS, is significantly decreased in HCC and liver cancer cell lines compared to immortalized normal cell lines and adjacent non-tumor tissues [28]. These results indicated that ROS may be involved in lincRNA-p21-induced ER stressmediated apoptosis.

\section{ER stress contributes to lincRNA-p21 effects on hepatocellular carcinoma}

The above results demonstrated that lincRNA-p21 regulated tumor growth, apoptosis and ER stress. One question remained unknown was whether ER stress accounted for lincRNA-p21-induced apoptosis and growth arrest. Therefore, we inhibited ER stress with salubrinal, a reported inhibitor for phosphorylation of eIF $2 \alpha$, in HepG2 cells (Figure 9A). Salubrinal treatment significantly rescued lincRNA-p21-induced apoptosis of HepG2 cells (Figure 9B). Significantly, salubrinal also inhibited lincRNA-p21-mediated growth inhibition of HepG2 cells in vitro and in vivo (Figure 9C and 9D). These findings demonstrated that ER stress, at least in part, contributes to lincRNA-p21-induced apoptosis and growth arrest.

\section{DISCUSSION}

Using in vitro and in vivo evidence, we demonstrate here that lincRNA-p21 serves as a suppressor for HCC development and drug resistance partly by activating ER stress. We first show the downregulation of lincRNA-p21 in patients with $\mathrm{HCC}$, which is correlated with higher disease stage and poor survival. And then we demonstrate that lincRNA-p21 inhibits growth of liver cancer cells in vitro and in vivo. Our mechanism analysis reveals that lincRNA-p21 activates ER stress and mediates sorafenib-induced ER stress, apoptosis and growth arrest. Finally, we provide evidence that 
Table 1: Correlative analysis of lincRNA-p21 levels with clinicopathological features

\begin{tabular}{|c|c|c|c|c|}
\hline Characteristic & Number $(N=70)$ & $\begin{array}{c}\text { lincRNA-P21 Low } \\
(N=38)\end{array}$ & $\begin{array}{l}\text { lincRNA-P21 High } \\
(N=32)\end{array}$ & $P$ Value \\
\hline Sex & & & & 0.4945 \\
\hline Male & 49 & 25 & 24 & \\
\hline Female & 21 & 13 & 18 & \\
\hline Grade & & & & 0.0166 \\
\hline 1 & 11 & 2 & 9 & \\
\hline 2 & 53 & 31 & 22 & \\
\hline 3 & 6 & 5 & 1 & \\
\hline Stage & & & & 0.0023 \\
\hline 1 & 41 & 16 & 25 & \\
\hline 2 or 3 & 29 & 22 & 7 & \\
\hline Multiple Tumors & & & & 0.5715 \\
\hline No & 39 & 20 & 19 & \\
\hline Yes & 31 & 18 & 13 & \\
\hline $\begin{array}{l}\text { Vascular Invasion } \\
\text { (Macro) }\end{array}$ & & & & 0.1903 \\
\hline No & 43 & 26 & 17 & \\
\hline Yes & 27 & 12 & 15 & \\
\hline $\begin{array}{l}\text { Vascular Invasion } \\
\text { (Micro) }\end{array}$ & & & & 0.0209 \\
\hline No & 29 & 11 & 18 & \\
\hline Yes & 41 & 27 & 14 & \\
\hline $\mathrm{HBV}$ & & & & 0.7434 \\
\hline No & 12 & 6 & 6 & \\
\hline Yes & 58 & 32 & 26 & \\
\hline Cirrhosis & & & & 0.1613 \\
\hline No & 33 & 15 & 18 & \\
\hline Yes & 37 & 23 & 14 & \\
\hline
\end{tabular}

lincRNA-p21 effects on liver cancer apoptosis and growth partly depends upon induction of ER stress.

LincRNA-p 21 is directly induced by 553 to play a critical role in the p53 transcriptional responses [15]. Both protein and mRNA targets for lincRNA-p21 have been identified $[15,29]$. Previous reports showed the involvement of lincRNA-p21 in cell cycle [19], Warburg effect [16], and cell survival [17], and these properties implicating functional roles of lincRNA-p21 in carcinoma. Chou et al. [30] reported the involvement of lincRNA-p21 in mammary cancer cells through control of HuR/elavL1 expression. In colon cancer tissues, lincRNA-p21 is significantly down-regulated [31, 32]. In human HCC, lincRNA-p21 level was also down-regulated.
The expression level of lincRNA-p21 was negatively correlated with AFP serum level, and low lincRNA-p21 level predicted poor overall and disease-free survival. Therefore, lincRNA-p21 could serve as a prognostic factor for favorable outcome. We used three HCC cell lines to study the function of lincRNA-p21 in vitro and in vivo. Both loss-of-function and gain-of-function experiments provided evidence that lincRNA-p21 was a tumor suppressor lincRNA, and treatments that activate lincRNA-p21 may be promising for HCC therapy.

Here we identified a new biological process that lincRNA-p21 participates in. We found that lincRNA-p21 could activate ER stress by increasing the expression of IRE1, CHOP and GRP78. However, in the reported targets 


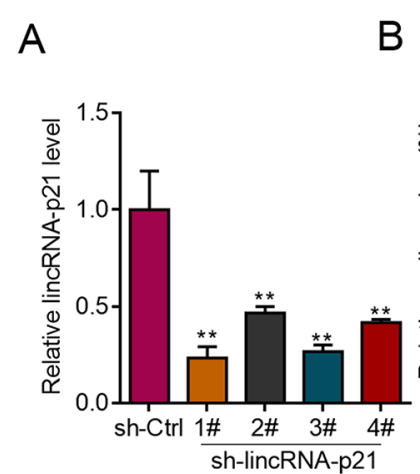

E

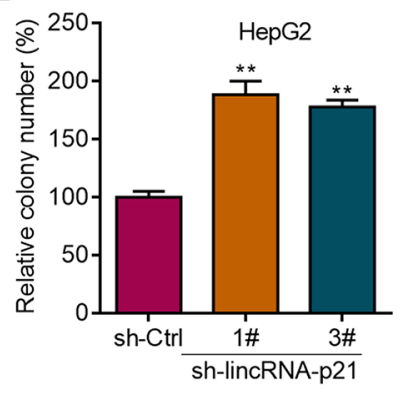

B

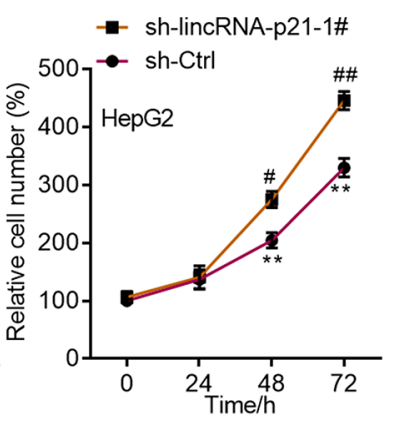

$\mathrm{F}$

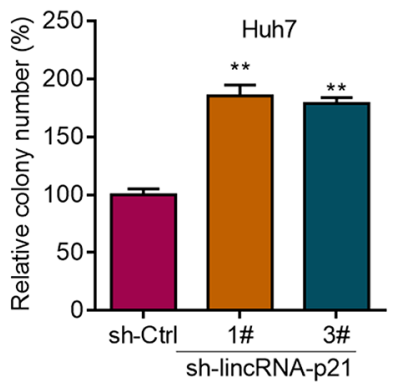

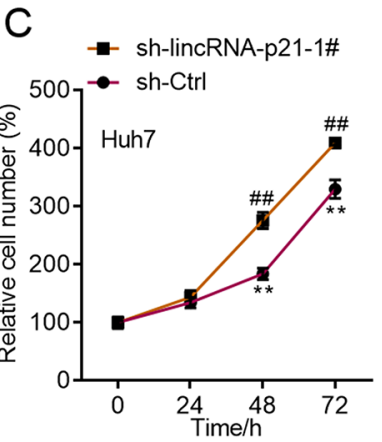

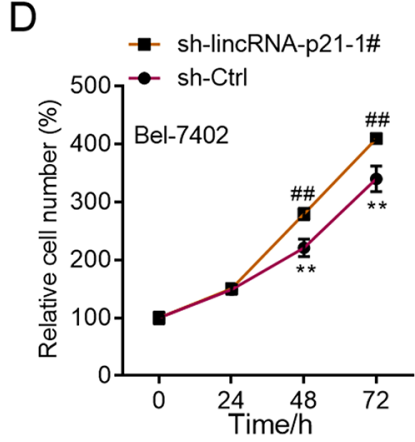

G

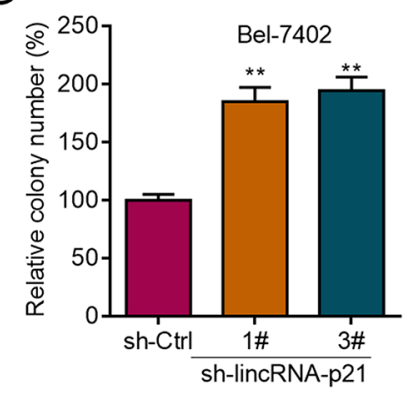

Figure 3: LincRNA-p21 knockdown facilitates proliferation and colony formation of liver cancer cells. A. Lentivirusmediated lincRNA-p21 knockdown by short hairpin RNAs (shRNAs) in HepG2 cells. B-D. LincRNA-p21 knockdown promotes proliferation of HepG2 (B), Huh7 (C), and Bel-7042 (D) cells. ${ }^{* *} p<0.01$ vs. sh-Ctrl 0 day; $\# p<0.05$ and \#\#p $<0.01$ vs. sh-Ctrl of the corresponding time points. E-G. LincRNA-p21 knockdown promotes colony formation of HepG2 (E), Huh7 (F), and Bel-7042 (G) cells. $* * p<0.01$ vs. sh-Ctrl.

A

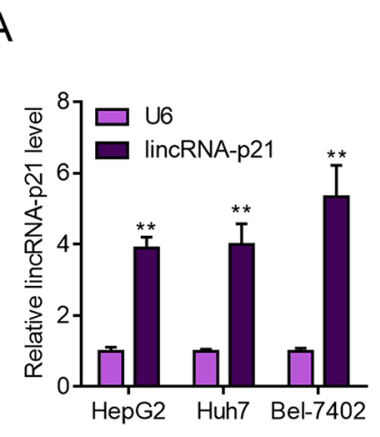

$E$

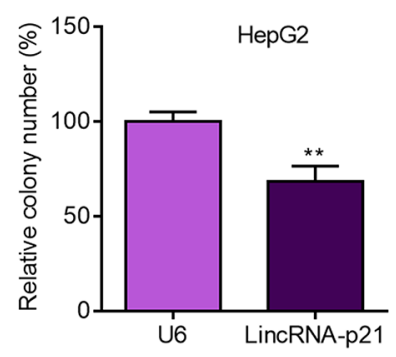

B

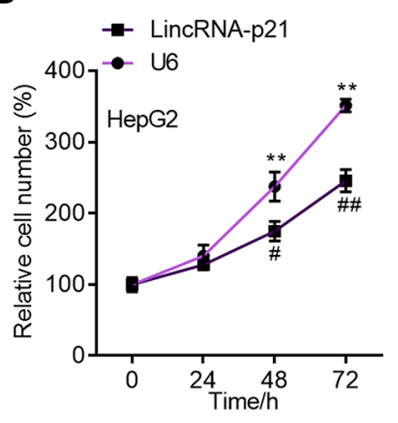

$\mathrm{F}$

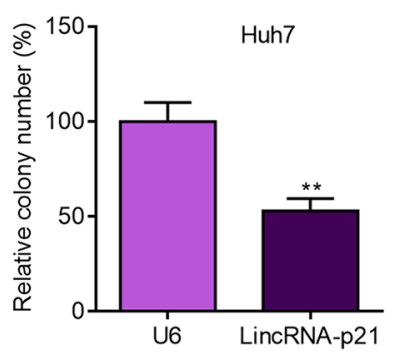

G
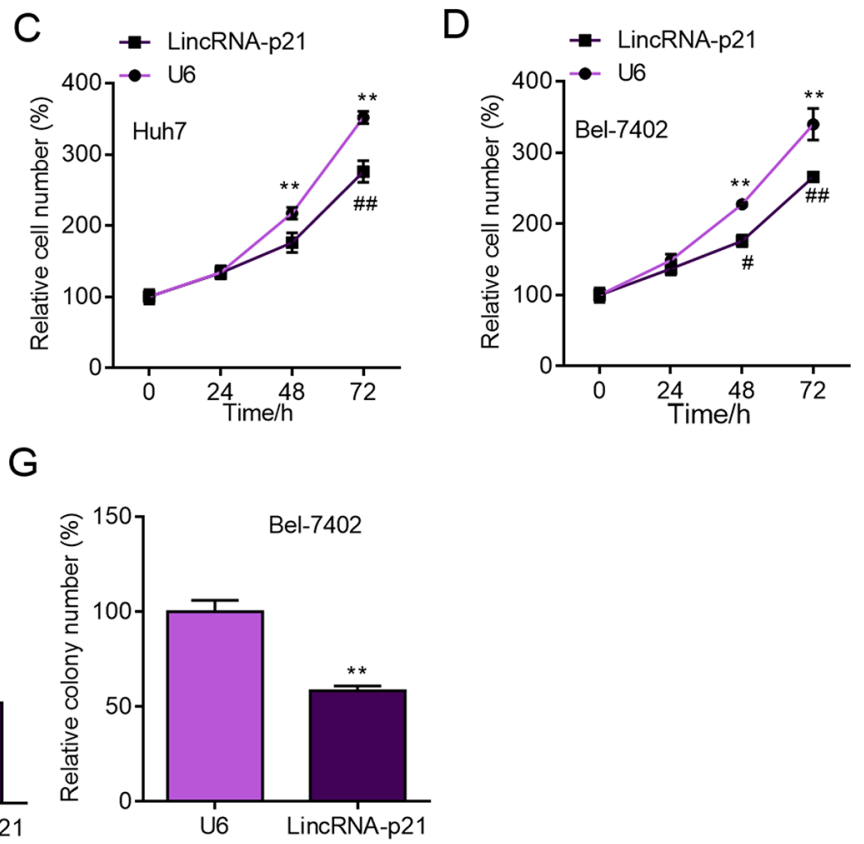

Figure 4: LincRNA-p21 overexpression inhibits liver cancer cell proliferation and colony formation. A. Lentivirusmediated lincRNA-p21 overexpression in HepG2, Huh7 and Bel-7402 cells. The level of lincRNA-p21 was checked 24 hours post lentivirus infection. ${ }^{* *} p<0.01$ vs. U6. $\$ \$ p<0.01$ vs. U6. \#\#p $<0.01$ vs. U6. $\& \& p<0.01$ vs. U6. B-D. LincRNA-p21 overexpression inhibits proliferation of HepG2 (B), Huh7 (C), and Bel-7402 (D) cells. * $p<0.05$ and $* * p<0.01$ vs U6 0 day; $\# p<0.05$ and \#\#p $<0.01$ vs U6 of the corresponding time points. E-G. LincRNA-p21 overexpression inhibits colony formation of HepG2 (E), Huh7 (F) and Bel-7402 (G) cells. ${ }^{* *} p<0.01$ vs U6. 
A

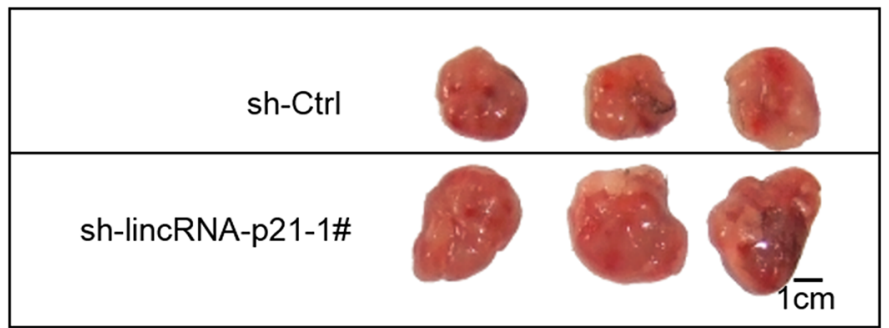

C

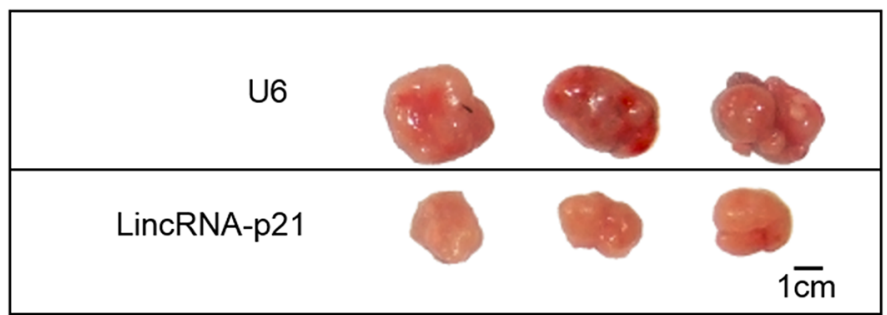

E

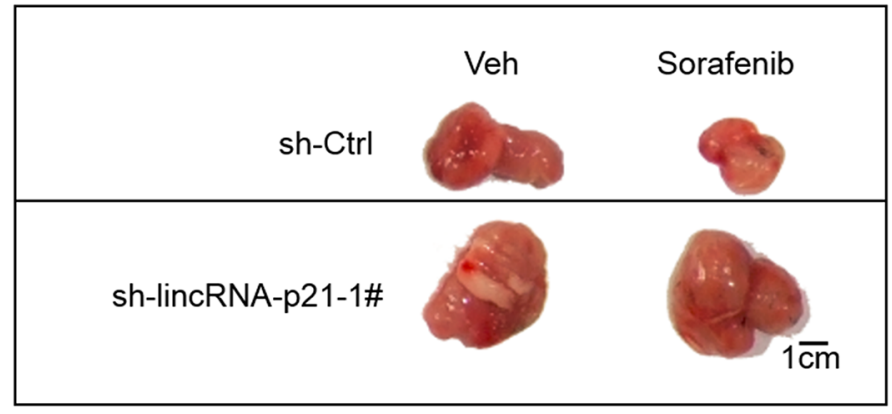

B

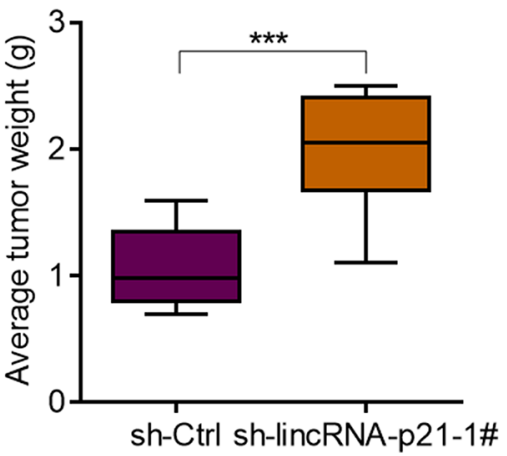

$\mathrm{D}$
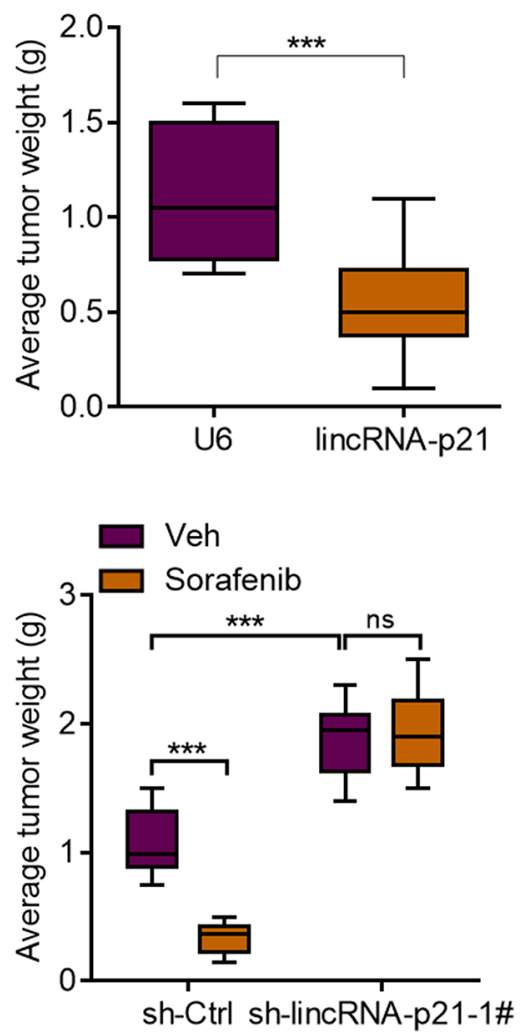

Figure 5: lincRNA-p21 regulates hepatocarcinoma growth in vivo. A. Representative photograph showing tumor size of shCtrl and sh-LincRNA-p21 groups. HepG2 cells were infected with lentivirus-mediated sh-Ctrl or sh-LincRNA-p21 and tumor xenograft experiment was performed. The mice were sacrificed four weeks after tumor implantation. B. Quantitative data for tumor weight in sh-Ctrl and sh-lincRNA-p21 groups. $N=10$ in each group. C. Representative photograph showing tumor size of U6 and lincRNA-p21 groups. HepG2 cells were infected with lentivirus overexpressing U6 or human lincRNA-p21 and tumor xenograft experiment was performed. The mice were sacrificed four weeks after tumor implantation. D. Quantitative data for tumor weight in U6 and lincRNA-p21 groups. $N=10$ in each group. E-F. LincRNA-p21 mediates sorafenib-induced inhibition of HepG2 cell growth in vivo. Sorafenib (30 mg/kg/d) was administered in $100 \mu \mathrm{L}$ by intraperitoneal injection on day three after tumor implantation. $N=10$ in each group in $(\mathrm{F}) * * * p<0.001$.

of lincRNA-p21, none of these genes were observed [15], indicating that lincRNA-p21 may regulate ER stress indirectly. These finding identified new biological function of lincRNA-p21 other than metabolism, and cell reprogramming $[15,16,19,29]$. However, how lincRNA-p21 regulates ER stress remains to be further explained.

Sorafenib is a clinical drug for HCC [25]. Previous reports indicated that sorafenib induce apoptosis and growth arrest of HCC cells partly through activating ER stress [5-9]. However, the underlying mechanism remains largely unexplored. We identified here lincRNA-p21 contributed to sorafenib-induced ER stress and apoptosis. Knockdown of lincRNA-p21 blocked sorafenib effects on ER stress, apoptosis and growth inhibition, indicating lincRNA-p21 low expression is one of the reasons for drug resistance in HCC and high level of lincRNA-p21 may predict favorable outcome post sorafenib therapy. We also found that sorafenib induced expression of p53 downstream genes, Puma, Mdm2, Noxa and Bax, which was blocked by lincRNA-p21 knockdown. Therefore, lincRNA-p21 may 

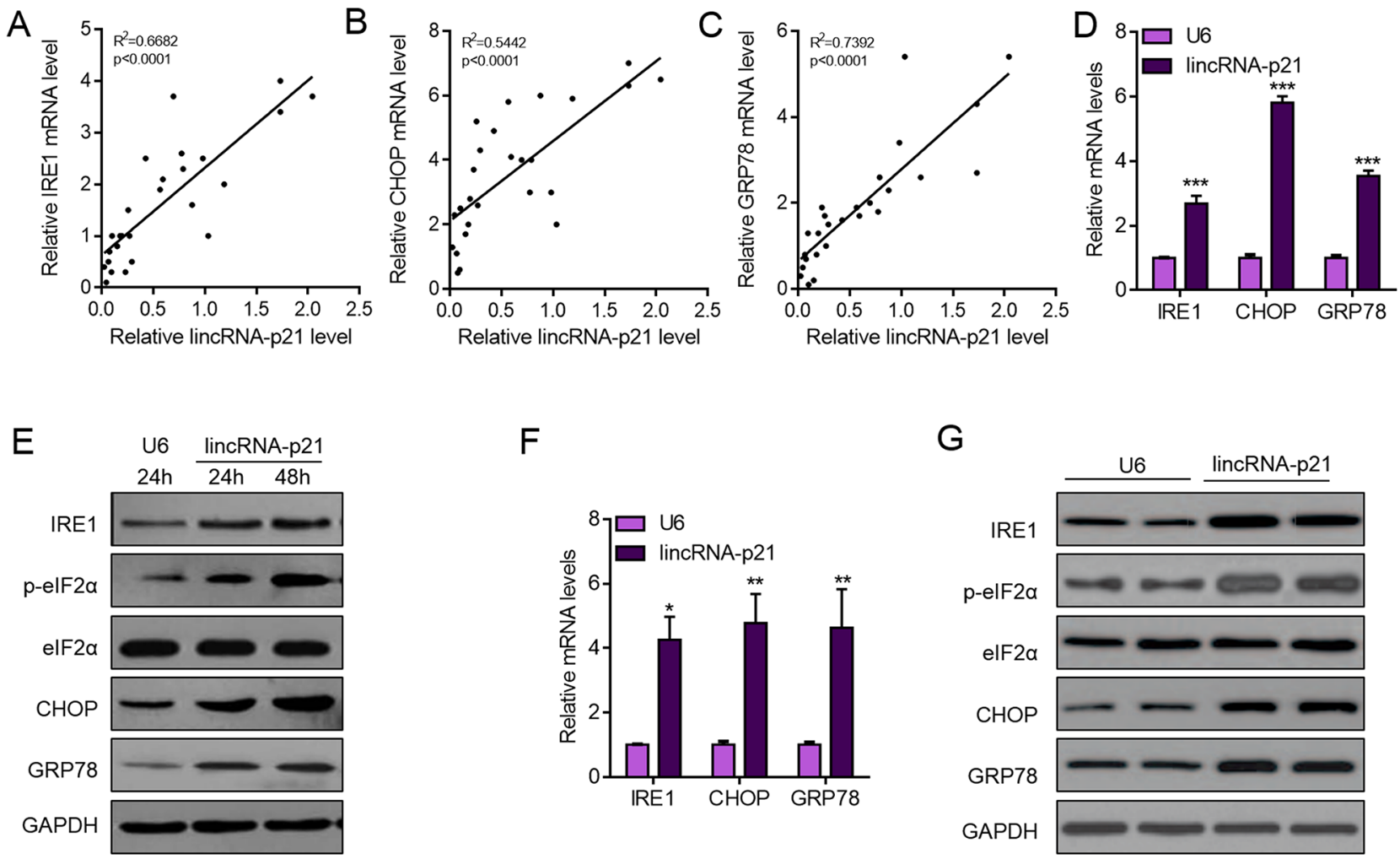

Figure 6: LincRNA-p21 overexpression activates ER stress in hepatocarcinoma. A-C. LincRNA-p21 level is positively correlated with mRNA levels of ER stress markers (IRE1, CHOP, and GRP78) in human hepatocellular carcinoma. $N=26$ in each group. D. LincRNA-p21 overexpression promotes the mRNA levels of ER stress markers (IRE1, CHOP, and GRP78) in HepG2 cells. HepG2 cells were infected with lentivirus overexpressing U6 or lincRNA-p21 for 48 hours. ${ }^{* *} p<0.001 v s$. U6. E. Western blot showing lincRNA-p21 promotes the activation of ER stress in HepG2 cells. F. LincRNA-p21 overexpression facilitates the mRNA levels of ER stress markers in vivo. Tumor xenograft experiment was performed using HepG2 cells. The mice were sacrificed four weeks after tumor implantation. ${ }^{*} p<0.05$ and $* * p<0.01$ vs. U6. $N=5$ in each group. G. Representative western blot showing lincRNA-p21 promotes the activation of ER stress in vivo. Tumor xenograft experiment was performed using HepG2 cells. The mice were sacrificed four weeks after tumor implantation.

also target p53 to induce apoptosis as previously reported [17]. Previous reports show that lincRNA-p21 increases following X-ray treatment, and enforced expression of the lincRNA enhances the sensitivity of radiotherapy for CRC by promoting cell apoptosis [32]. These findings implicate that up-regulation of lincRNA-p21 may contribute to drug treatment and radiotherapy.

In conclusion, we identify lincRNA-p21 as a suppressor for HCC growth and drug resistance through ER stress, and further explain how sorafenib regulates ER stress and HCC development. LincRNA-p21 may serve as a prognostic factor for outcome and drug resistance.

\section{MATERIALS AND METHODS}

\section{Patients and tissue specimens}

70 cases of hepatocellular carcinoma (HCC) patients with full case history between May 2001 and October 2007 were enrolled in the present study. Fresh HCC or adjacent normal liver tissues were obtained and stored at $-80^{\circ} \mathrm{C}$ before use. The patients were recruited at Eastern
Hepatobiliary Surgery Hospital, Second Military Medical University (Shanghai, China). All HCC samples were histopathologically re-evaluated independently by two pathologists before further analysis. Healthy liver samples were obtained from donor livers used for transplantation that were pathologically evaluated before transplantation. Further patient information is included in Table 1. A written form of informed consent was obtained from all patients and donors before any treatment and experiments. The study was approved by the Clinical Research Ethics Committee of Second Military Medical University. The methods were carried out in accordance with the approved guidelines.

\section{Quantitative real-time PCR (q-PCR)}

Total RNA was extracted from cells or fresh tissues with TRIzol (Invitrogen \# 10296010). cDNA was synthesized from two $\mu \mathrm{g}$ of total RNA with One-Step RT-PCR Kit (TaKaRa \# RR086A). Then, q-PCR was performed with the SYBR Green (TaKaRa \# DRR420A) on an ABI-7500 RT-PCR system (Applied Biosystems). The primers were listed in Supplemental Table S1. 
A

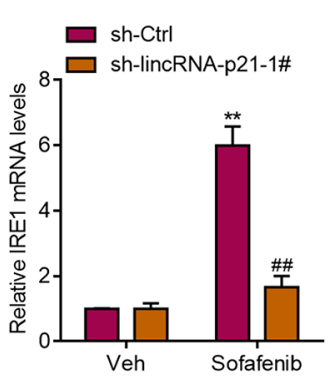

$\mathrm{E}$

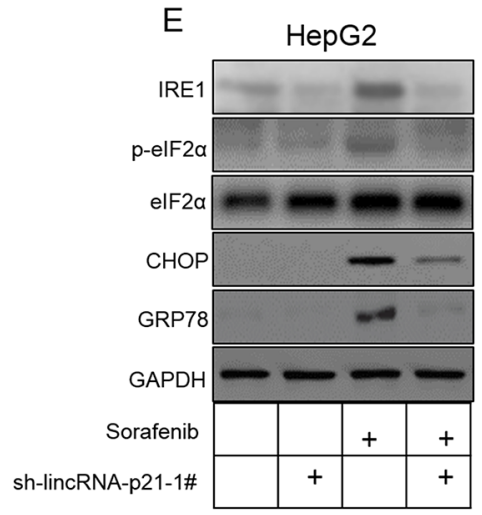

B

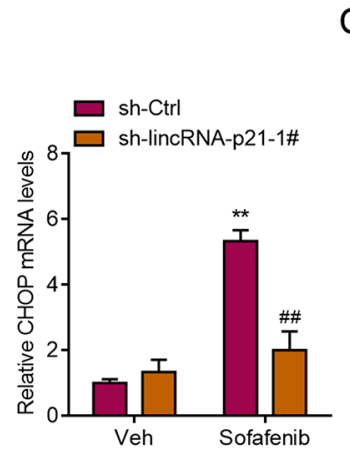

C
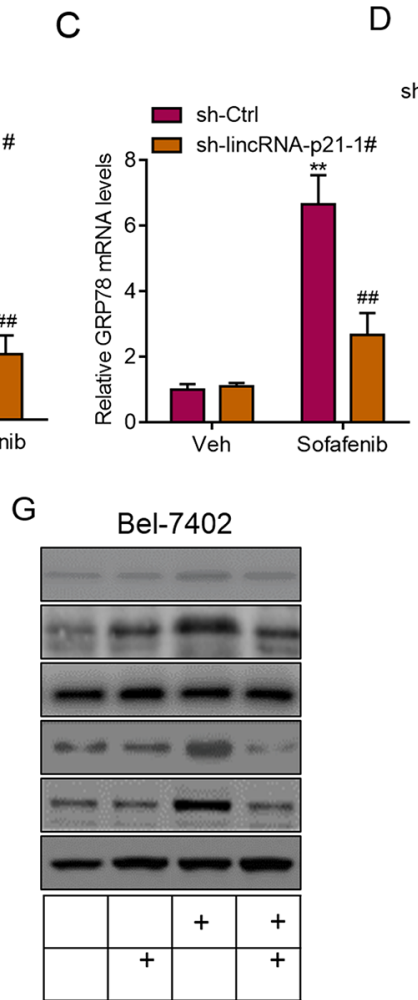

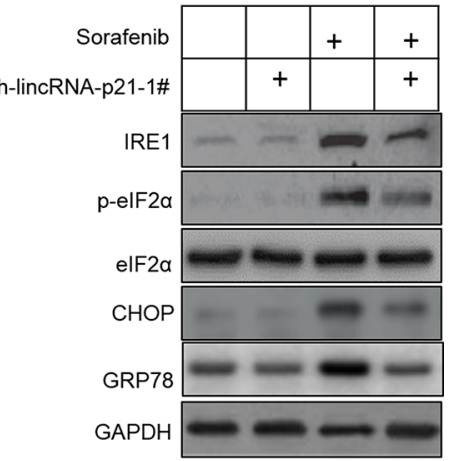

Figure 7: LincRNA-p21 contributes to sorafenib-induced ER stress in vivo. A-C. LincRNA-p21 knockdown inhibits sorafenib-induced expression of ER stress markers in vivo. Tumor xenograft experiment was performed using HepG2 cells. The mice were sacrificed four weeks after tumor implantation. Sorafenib $(30 \mathrm{mg} / \mathrm{kg} / \mathrm{d})$ was administered in $100 \mu \mathrm{L}$ by intraperitoneal injection on day three after tumor implantation. ${ }^{* *} p<0.01$ vs. sh-Ctrl + Vehicle (Veh); \#\#p<0.01 vs. sh-Ctrl + sorafenib. $N=5$ in each group. D. Representative western blot showing lincRNA-p21 knockdown blocks sorafenib-induced activation of ER stress in vivo. Tumor xenograft experiment was performed using HepG2 cells. The mice were sacrificed four weeks after tumor implantation. E-G. Representative western blot showing lincRNA-p21 knockdown blocks sorafenib-induced activation of ER stress in HepG2 (E), Huh7 (F), and Bel-7402 (G) cells. Cells were infected with lentivirus carrying sh-lincRNA-p21 or ctrl shRNA for 24 hours followed by $20 \mu \mathrm{M}$ sorafenib for 48 hours.

\section{Cell culture and retroviral transduction}

The liver cancer cell lines HepG2, Huh7, Hep3B, LM9, Bel-7402 and SMMC-7721 were cultured as described previously [33]. Normal human hepatocytes were isolated from specimens obtained from patients undergoing hepatic resections for the therapy of hepatic tumors after informed consent and according to the rules of the ethics committee of the Second Military Medical University. The liver cancer cells and normal human hepatocytes were cultured in high glucose-containing Dulbecco's modified Eagle's medium (DMEM) supplemented with 10\% FBS, $100 \mathrm{units} / \mathrm{ml}$ penicillin and $100 \mu \mathrm{g} / \mathrm{ml}$ streptomycin.

Sh-lincRNA-p21 and control shRNA (sh-Ctrl) lentivirus particles were purchased from GenePharma. The shRNA sequences targeting lincRNA-p21 is shown in Supplementary Table S2. Lentivirus expressing human lincRNA-p21 was generated by sub-cloning mouse lincRNA-p21 cDNA to the pSLIK lentivirus expression system (cloning primer forward: TGGCAGTCTGACCCACACTCCCCACGCCC; reverse: ACAGTGCACAGACAATCATACACACGTGT). For retroviral packaging, 293T cells were co-transfected with the retroviral particles. For transduction, cells were incubated with virus-containing supernatant in the presence of $8 \mathrm{mg} / \mathrm{ml}$ polybrene. After 48 hours, infected cells were selected for 72 hours with puromycin $(2 \mathrm{mg} / \mathrm{ml})$ or hygromycin $(200 \mathrm{mg} / \mathrm{ml})$.

\section{Cell proliferation assay}

Cell proliferation ability of the liver cancer cells was monitored by CCK-8 Cell Proliferation/Viability Assay Kit (Sigma \# 96992) in according to the guidelines.

\section{Colony formation}

For liver cancer cell colony formation assay, liver cancer cells were suspended in $1.5 \mathrm{ml}$ complete medium supplemented with $0.45 \%$ low melting point agarose (Invitrogen \# 16520-050). The cells were placed in $35 \mathrm{~mm}$ tissue culture plates containing $1.5 \mathrm{ml}$ complete medium and agarose $(0.75 \%)$ on the bottom layer. The cells were cultured for 14 days. Cell colonies were stained with $0.005 \%$ crystal violet and analyzed using a microscope. The colony number in each well was calculated. 
A
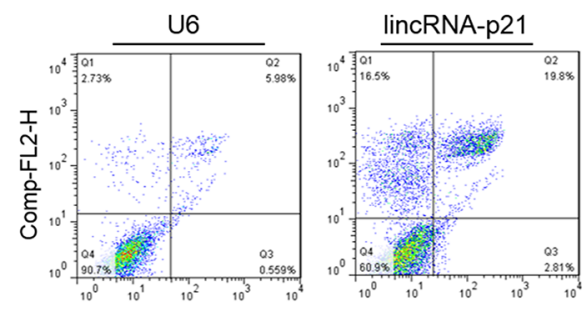

B

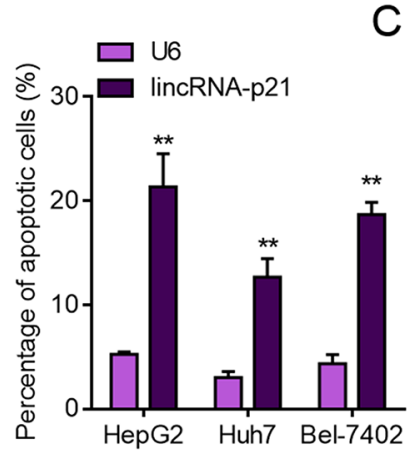

C

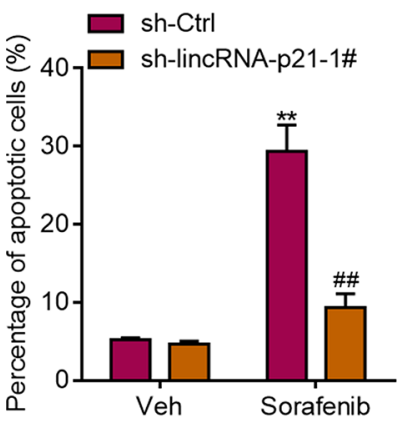

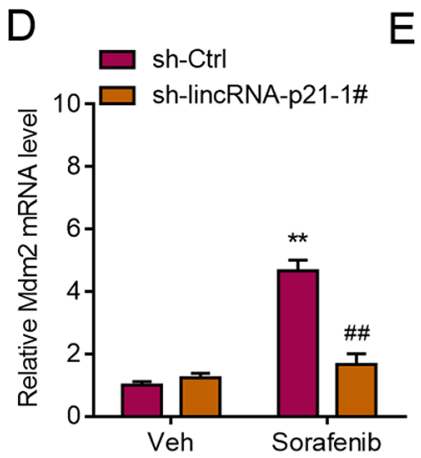
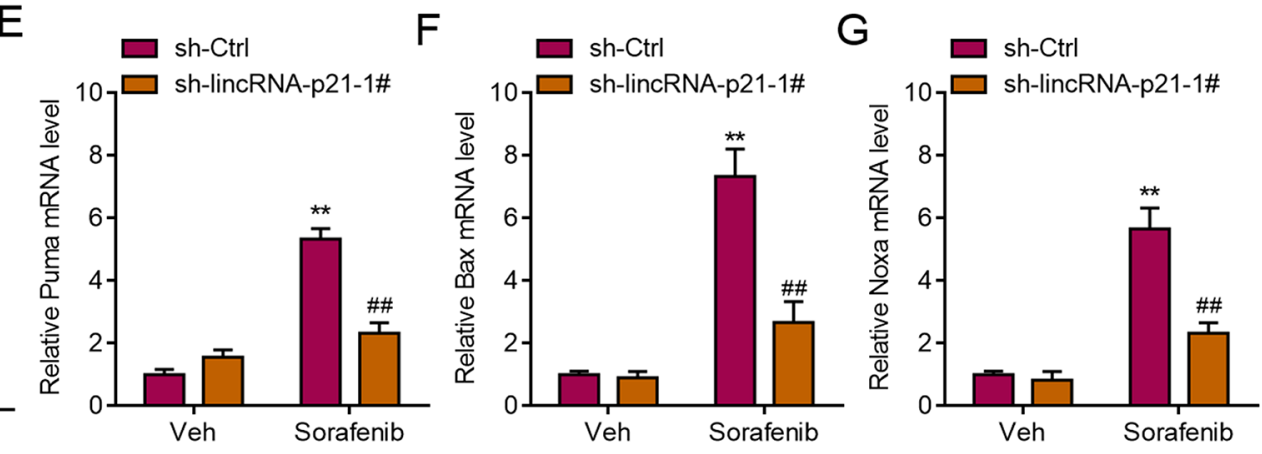

Figure 8: LincRNA-p21 regulates ER stress-related apoptosis. A. Representative FACS results showing lincRNA-p21 overexpression promotes apoptosis of HepG2 cells. B. Quantitative data showing lincRNA-p21 induces apoptosis of HepG2, Huh7, and Bel-7042 cells. Cells were infected with lentivirus overexpressing U6 or lincRNA-p21 for 48 hours. ${ }^{*} p<0.01 v s$. U6. C. LincRNA-p21 knockdown blocks sorafenib-induced apoptosis of HepG2 cells. Cells were infected with lentivirus carrying sh-lincRNA-p21 or ctrl shRNA for 24 hours followed by $20 \mu \mathrm{M}$ sorafenib for 48 hours. ${ }^{* *} p<0.01 v s$. sh-Ctrl + Vehicle (Veh); \#\#p<0.01 vs. sh-Ctrl+sorafenib. D-G. LincRNA-p21 knockdown inhibits expression of sorafenib-induced expression of p53 downstream markers (Mdm2, Puma, Bax and Noxa). Cells were infected with lentivirus carrying sh-lincRNA-p21 or ctrl shRNA for 24 hours followed by $20 \mu \mathrm{M}$ sorafenib for 48 hours. $* * p<0.01 v s$. sh-Ctrl +Vehicle (Veh); \#\#p $<0.01$ vs. sh-Ctrl+sorafenib.

\section{Tumor xenograft experiments}

Equal numbers of HepG2 cells stably expressing either control or lincRNA-p21 knockdown, U6 or lincRNA-p21 overexpression vectors $\left(5 \times 10^{6}\right)$ in $100 \mu$ l of a 1:1 mixture of culture medium and growth factorreduced Matrigel were implanted subcutaneously into the forelegs of 4- to 5-week-old male BALB/c athymic $\mathrm{nu} / \mathrm{nu}$ mice (Vital River). When the tumors reached approximately $7-10 \mathrm{~mm}$ in diameter, they were prepared to form a brei and then injected subcutaneously into nude mice. Tumor growth was monitored by tumor weight at the end of study (Four weeks for in vivo tumor growth). The study was approved by the Animal Research Ethics Committee of Second Military Medical University. The methods were carried out in accordance with the approved guidelines.

\section{Western blot}

Cells or fresh tissues were lysed in RIPA lysis buffer with mixture of protease inhibitors (Beyotime \#ST506) and PhosSTOP (Roche \#04906845001). $30 \mu \mathrm{g}$ total proteins were subjected to $12 \%$ SDS- polyacrylamide gel. After electrophoresis, the proteins were transferred to PVDF membranes, which were then blocked with $5 \%$ milk for 2 hours. The membranes were then probed with primary antibody for IRE1 (Abcam \# ab37073), CHOP (Cell Signaling Technology \# 2895), GAPDH (Santa Cruz \#sc32233), GRP78 (Abcam \# ab32618), p-eIF2 $\alpha$ (Cell Signaling Technology \# 9721), eIF2 $\alpha$ (Cell Signaling Technology \# 3597), DUOX1 (Abcam \#ab78919), p-PERK (Cell Signaling Technology \#3179), PERK (Cell Signaling Technology \#3192) at $4^{\circ} \mathrm{C}$ overnight, and then the membranes were washed with TBST and incubated with HRP-conjugated secondary antibodies (Santa Cruz \# sc-2030) for 1.5 hours and finally washed and visualized using Chemiluminescent ECL reagent (Beyotime \# P0018).

\section{Apoptosis assay}

Apoptosis of liver cancer cells was evaluated with fluorescence-activated cell sorting (FACS) assay. FACS analysis was conducted with an Annexin V-FITC Apoptosis Detection Kit (Abcam \# ab14086) according to the manufacturer's protocol. A FACS Calibur flow cytometer was used for data analysis. 
A

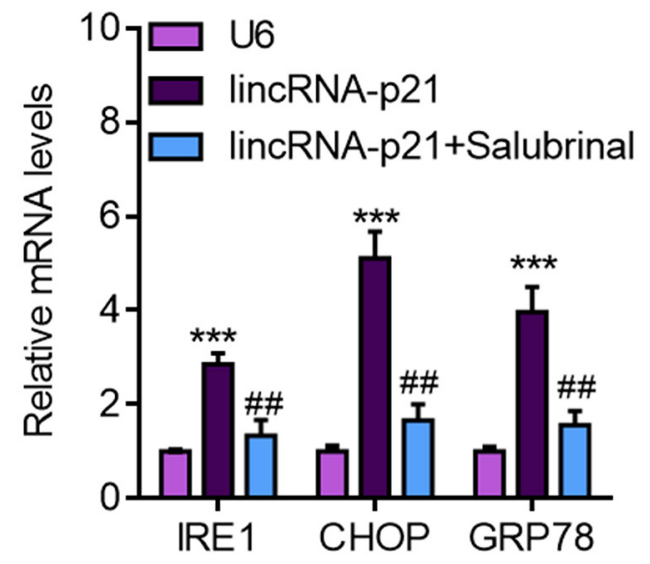

C

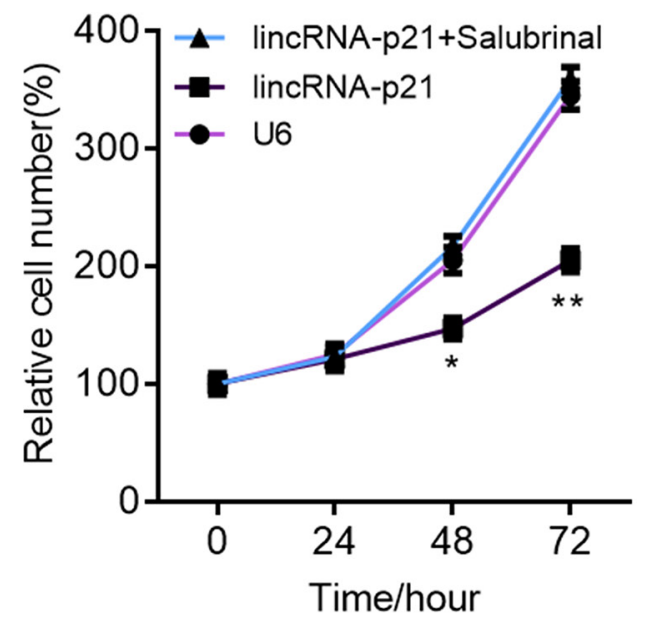

B

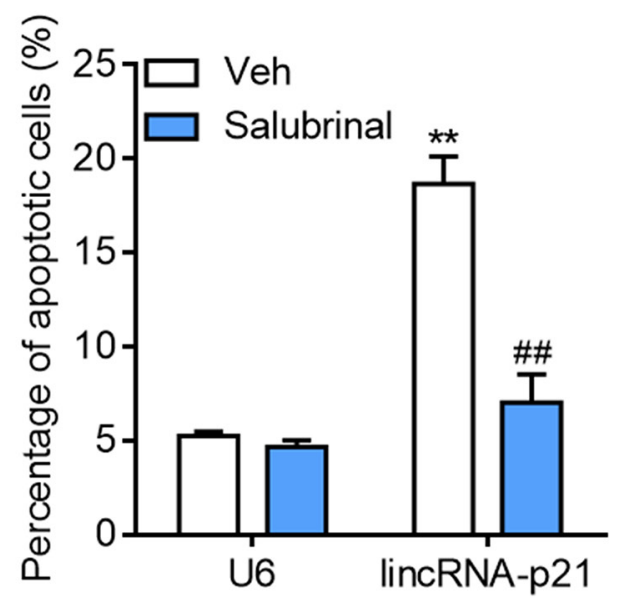

D

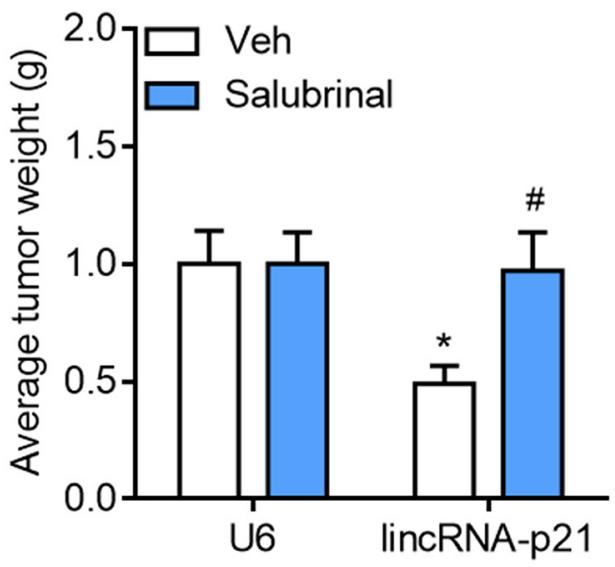

Figure 9: Inhibition of ER stress blocks lincRNA-p21 functions in hepatocarcinoma. A-B. The cells were infected with indicated lentivirus for 24 hours followed by salubrinal $(10 \mu \mathrm{M})$ for another 24 hours. (A) Inhibition of ER stress with salubrinal $(10 \mu \mathrm{M})$ inhibits lincRNA-p21 induced expression of ER stress markers in HepG2 cells. ${ }^{* * *} p<0.001 v s$. U6; \#\# $<0.01 v s$. lincRNA-p21. (B) Salubrinal reduces lincRNA-p21-induced apoptosis in HepG2 cells. ${ }^{* *} p<0.01 v s$. U6+Vehicle (Veh); \#\#p $<0.01 v s$. lincRNA-p21+Vehicle (Veh). C. Salubrinal inhibition blocks lincRNA-p21-induced repression of HepG2 cell proliferation. The cells were infected with indicated lentivirus with/without salubrinal $(10 \mu \mathrm{M})$ for indicated times. ${ }^{*} p<0.05$ and ${ }^{* *} p<0.01 v s$. U6 of the same time points. D. Salubrinal (1 mg/ $\mathrm{kg} / \mathrm{d}$ ) inhibits lincRNA-p21-induced repression of HepG2 cell growth in vivo. Tumor xenograft experiment was performed using HepG2 cells. The mice were sacrificed four weeks after tumor implantation. ${ }^{*} p<0.05 v s$. U6+Vehicle (Veh); \#p $<0.05 v s$. lincRNA-p21+Vehicle (Veh). $N=10$ in each group.

\section{ROS determination}

Dihydroethidium (DHE, Invitrogen \#D11347) was used for detecting ROS generation in cardiomyocytes as per the manufacturer's protocol.

\section{Statistics}

Values were expressed as Mean \pm SEM. Statistical differences between two groups were determined using unpaired or paired Student's $t$ test. For more than two groups, one-way or two-way ANOVA analysis were applied. The correlation of lincRNA-p21 levels with patients' clinicopathological variables was analyzed by the $\chi 2$ test or Fisher's exact test. The Kaplan-Meier method was used to estimate overall and disease-free survival. Survival differences according to lincRNA-p21 expression were analyzed by the log-rank test. Linear regression analysis was performed to analyze the relation between lincRNA-p21 level and AFP serum level as well as ER stress markers in patients with HCC. $P$ values of less than 0.05 were considered statistically significant. 


\section{CONFLICTS OF INTEREST}

None.

\section{GRANT SUPPORT}

This work was supported by Grant 81372355 from the National Natural Science Foundation and Grant 2012ZX10002-017 from the National Science and Technology Major Project of China.

\section{REFERENCES}

1. Farazi P.A., DePinho R.A. Hepatocellular carcinoma pathogenesis: from genes to environment. Nat. Rev. Cancer. 2006; 6:674-687.

2. Poon R.T. Prevention of recurrence after resection of hepatocellular carcinoma: a daunting challenge. Hepatology. 2011; 54:757-759.

3. Avila M., Berasain C. Making Sorafenib Irresistible: In Vivo Screening for Mechanisms of Therapy Resistance in Hepatocellular Carcinoma Hits on Mapk14. Hepatology. 2015; 61:1755-1757.

4. Moenner M., Pluquet O., Bouchecareilh M., Chevet E. Integrated Endoplasmic Reticulum Stress Responses in Cancer. Cancer Res. 2007; 67:10631-10634.

5. Hetz C., Chevet E., Harding H.P. Targeting the unfolded protein response in disease, Nat Rev. Drug Discov. 2013; 12:703-719.

6. Shuda M., Kondoh N., Imazeki N., Tanaka K., Okada T., Mori K., Hada A., Arai M., Wakatsuki T., Matsubara O., Yamamoto N., Yamamoto M. Activation of the ATF6, XBP1 and grp78 genes in human hepatocellular carcinoma: a possible involvement of the ER stress pathway in hepatocarcinogenesis. J. Hepatol. 2003; 38:605-614.

7. Nakagawa H., Umemura A., Taniguchi K., Font-Burgada J., Dhar D., Ogata H., Zhong Z., Valasek Mark A., Seki E., Hidalgo J., Koike K., Kaufman Randal J., Karin M. ER Stress Cooperates with Hypernutrition to Trigger TNFDependent Spontaneous HCC Development. Cancer Cell. 2014; 26:331-343.

8. Shi Y.H., Ding Z.B., Zhou J., Hui B., Shi G.M., Ke A.W., Wang X.Y., Dai Z., Peng Y.F., Gu C.Y., Qiu S.J., Fan J. Targeting autophagy enhances sorafenib lethality for hepatocellular carcinoma via ER stress-related apoptosis. Autophagy. 2011; 7:1159-1172.

9. Yi P., Higa A., Taouji S., Bexiga M.G., Marza E., Arma D., Castain C., Le Bail B., Simpson J.C., Rosenbaum J., Balabaud C., Bioulac-Sage P., Blanc J.-F., Chevet E. Sorafenib-Mediated Targeting of the AAA+ ATPase p97/VCP Leads to Disruption of the Secretory Pathway, Endoplasmic Reticulum Stress, and Hepatocellular Cancer Cell Death, Mol. Cancer Ther. 2012; 11:2610-2620.
10. Chiang P.-C., Chien C.-L., Pan S.-L., Chen W.-P., Teng C.M., Shen Y.-C., Guh J.-H. Induction of endoplasmic reticulum stress and apoptosis by a marine prostanoid in human hepatocellular carcinoma. J. Hepatol. 2005; 43:679-686.

11. Cheng C.Y., Lin Y.H., Su C.C. Curcumin inhibits the proliferation of human hepatocellular carcinoma $\mathrm{J} 5$ cells by inducing endoplasmic reticulum stress and mitochondrial dysfunction. Int. J. Mol. Med. 2010; 26:673-678.

12. Yeh T.C., Chiang P.C., Li T.K., Hsu J.L., Lin C.J., Wang S.W., Peng C.Y., Guh J.H. Genistein induces apoptosis in human hepatocellular carcinomas via interaction of endoplasmic reticulum stress and mitochondrial insult. Biochem. Pharmacol. 2007; 73:782-792.

13. Yang X., Xie X., Xiao Y.-F., Xie R., Hu C.-J., Tang B., Li B.-S., Yang S.-M. The emergence of long non-coding RNAs in the tumorigenesis of hepatocellular carcinoma. Cancer Lett. 2015; 360:119-124.

14. Takahashi K., Yan I., Haga H., Patel T. Long noncoding RNA in liver diseases. Hepatology. 2014; 60:744-753.

15. Huarte M., Guttman M., Feldser D., Garber M., Koziol M.J., Kenzelmann-Broz D., Khalil A.M., Zuk O., Amit I., Rabani M., Attardi L.D., Regev A., Lander E.S., Jacks T., Rinn J.L. A Large Intergenic Noncoding RNA Induced by p53 Mediates Global Gene Repression in the p53 Response. Cell. 2010; 142:409-419.

16. Yang F., Zhang H., Mei Y., Wu M. Reciprocal regulation of HIF-1alpha and lincRNA-p21 modulates the Warburg effect. Mol. Cell. 2014; 53:88-100.

17. Wu G., Cai J., Han Y., Chen J., Huang Z.P., Chen C., Cai Y., Huang H., Yang Y., Liu Y., Xu Z., He D., Zhang X., Hu X., Pinello L., Zhong D., He F., Yuan G.C., Wang D.Z., Zeng C. LincRNA-p21 regulates neointima formation, vascular smooth muscle cell proliferation, apoptosis, and atherosclerosis by enhancing p53 activity. Circulation. 2014; 130:1452-1465.

18. Bao X., Wu H., Zhu X., Guo X., Hutchins A.P., Luo Z., Song H., Chen Y., Lai K., Yin M., Xu L., Zhou L., Chen J., Wang D., Qin B., Frampton J., Tse H.F., Pei D., Wang H., Zhang B., Esteban M.A. The p53-induced lincRNA-p21 derails somatic cell reprogramming by sustaining $\mathrm{H} 3 \mathrm{~K} 9 \mathrm{me} 3$ and $\mathrm{CpG}$ methylation at pluripotency gene promoters. Cell Res. 2015; 25:80-92.

19. Dimitrova N., Zamudio J.R., Jong R.M., Soukup D., Resnick R., Sarma K., Ward A.J., Raj A., Lee J.T., Sharp P.A., Jacks T. LincRNA-p21 activates p21 in cis to promote Polycomb target gene expression and to enforce the G1/S checkpoint. Mol. Cell. 2014; 54:777-790.

20. Quagliata L., Matter M.S., Piscuoglio S., Arabi L., Ruiz C., Procino A., Kovac M., Moretti F., Makowska Z., Boldanova T., Andersen J.B., Hämmerle M., Tornillo L., Heim M.H., Diederichs S., Cillo C., Terracciano L.M. Long noncoding RNA HOTTIP/HOXA13 expression is associated with disease progression and predicts outcome in hepatocellular carcinoma patients. Hepatology. 2014; 59:911-923. 
21. Lai M.-c., Yang Z., Zhou L., Zhu Q.-q., Xie H.-y., Zhang F., Wu L.-m., Chen L.-m., Zheng S.-s. Long non-coding RNA MALAT-1 overexpression predicts tumor recurrence of hepatocellular carcinoma after liver transplantation. Med. Oncol. 2012; 29:1810-1816.

22. Panzitt K., Tschernatsch M.M.O., Guelly C., Moustafa T., Stradner M., Strohmaier H.M., Buck C.R., Denk H., Schroeder R., Trauner M., Zatloukal K. Characterization of HULC a Novel Gene With Striking Up-Regulation in Hepatocellular Carcinoma, as Noncoding RNA. Gastroenterology. 2007; 132:330-342.

23. Matouk I.J., DeGroot N., Mezan S., Ayesh S., Abu-lail R., Hochberg A., Galun E. The H19 Non-Coding RNA Is Essential for Human Tumor Growth. PLoS One. 2007; 2:e845.

24. Braconi C., Valeri N., Kogure T., Gasparini P., Huang N., Nuovo G.J., Terracciano L., Croce C.M., Patel T. Expression and functional role of a transcribed noncoding RNA with an ultraconserved element in hepatocellular carcinoma. Proc. Natl. Acad. Sci. U.S.A. 2011; 108:786-791.

25. Llovet J.M., Ricci S., Mazzaferro V., Hilgard P., Gane E., Blanc J.-F., de Oliveira A.C., Santoro A., Raoul J.-L., Forner A., Schwartz M., Porta C., Zeuzem S., Bolondi L., Greten T.F., Galle P.R., Seitz J.-F., Borbath I., Häussinger D., Giannaris T., Shan M., Moscovici M., Voliotis D., Bruix J. Sorafenib in Advanced Hepatocellular Carcinoma. New Engl. J. Med. 2008; 359:378-390.

26. Verfaillie T., Garg A.D., Agostinis P. Targeting ER stress induced apoptosis and inflammation in cancer. Cancer Lett. 2013; 332:249-264.

27. Liu L., Cao Y., Chen C., Zhang X., McNabola A., Wilkie D., Wilhelm S., Lynch M., Carter C. Sorafenib blocks the RAF/MEK/ERK pathway, inhibits tumor angiogenesis, and induces tumor cell apoptosis in hepatocellular carcinoma model PLC/PRF/5. Cancer Res. 2006; 66:11851-11858.

28. Ling Q., Shi W., Huang C., Zheng J., Cheng Q., Yu K., Chen S., Zhang H., Li N., Chen M. Epigenetic silencing of dual oxidase 1 by promoter hypermethylation in human hepatocellular carcinoma. Am. J. Cancer Res. 2014; 4:508-517.

29. Yoon J.-H., Abdelmohsen K., Srikantan S., Yang X., Martindale Jennifer L., De S., Huarte M., Zhan M., Becker Kevin G., Gorospe M. LincRNA-p21 Suppresses Target mRNA Translation. Mol. Cell. 2012; 47:648-655.

30. Chou S.D., Murshid A., Eguchi T., Gong J., Calderwood S.K. HSF1 regulation of [beta]-catenin in mammary cancer cells through control of HuR/elavL1 expression. Oncogene. 2015; 34:2178-2188.

31. Zhai H., Fesler A., Schee K., Fodstad Ø., Flatmark K., Ju J. Clinical Significance of Long Intergenic Noncoding RNAp21 in Colorectal Cancer. Clin. Colorectal Cancer. 2013; 12:261-266.

32. Wang G., Li Z., Zhao Q., Zhu Y., Zhao C., Li X., Ma Z., Li X., Zhang Y. LincRNA-p21 enhances the sensitivity of radiotherapy for human colorectal cancer by targeting the Wnt/ $\beta$-catenin signaling pathway. Oncol. Rep. 2014; 31:1839-1845.

33. Erdal E., Ozturk N., Cagatay T., Eksioglu-Demiralp E., Ozturk M. Lithium-mediated downregulation of PKB/Akt and cyclin $\mathrm{E}$ with growth inhibition in hepatocellular carcinoma cells. Int. J. Cancer. 2005; 115:903-910. 